\title{
EXCEPTIONAL INVARIANTS IN THE PARABOLIC INVARIANT THEORY OF CONFORMAL GEOMETRY
}

\author{
TOBY N. BAILEY AND A. ROD GOVER \\ (Communicated by Christopher Croke)
}

\begin{abstract}
We provide a construction for the exceptional invariants of certain modules for a parabolic subgroup of a pseudo-orthogonal group. The invariant theory of these modules has applications in conformal geometry.
\end{abstract}

\section{INTRODUCTION}

With [F], Fefferman initiated a program whereby certain sorts of geometric questions are reduced to problems in the invariant theory of modules for parabolic subgroups $P \subset G$ of semisimple Lie groups. Problems in the geometry of projective, conformal and CR structures can be approached in this way (see [Gr] for a review). In each case, the invariant theory problem is to list all the invariants of the relevant $P$-module-where by an invariant, we mean a $P$-equivariant polynomial map from the module to a 1 -dimensional $P$-module. Some invariants of the modules can be constructed as linear combinations of complete contractions of tensors. Such invariants are called Weyl invariants and the initial problem therefore is to determine to what extent all invariants are Weyl invariants. Fefferman [F] obtained partial answers to this question in the case that arises from the problem in CR geometry which he was considering, and also for a "model problem" (the modules $\mathscr{H}_{k}$ of $\S 2.1$ below) that turned out (see [EG]) to have an interesting interpretation in conformal geometry.

Recently Gover obtained a complete answer to a question of this sort pertaining to projective structures [Go] and following this, Bailey, Eastwood, and Graham [BEGr] solved both Fefferman's original problems and a similar problem (for the module $\mathscr{K}$ of $\S 2.2$ below) connected with invariants of conformal structures. Every invariant can be written as a sum of an odd invariant and an even invariant, where "odd" and "even" refer to behavior under orientation reversal. Also, every invariant can be written as a sum of invariants, each of which is homogeneous as a polynomial. For this reason, we consider only invariants that are odd or even, and homogeneous of some degree which we denote by $d$.

Received by the editors February 24, 1993 and, in revised form, November 2, 1993.

1991 Mathematics Subject Classification. Primary 53A55; Secondary 53A30, 32C16.

Key words and phrases. Parabolic invariant theory, conformal geometry, invariant differential operators, conformal invariants, exceptional invariants.

The authors are grateful for support from the Edinburgh Mathematical Society and the Australian Research Council. 
Invariants which are not Weyl invariants are termed exceptional. For the problems related to conformal geometry, it is shown in [BEGr] that for the modules $\mathscr{H}_{k}$ an invariant is exceptional if and only if it is odd and of degree $n$, and for $\mathscr{K}$ an invariant is exceptional if and only if it is odd and of degree $n / 2$ (and thus $\mathscr{K}$ has no exceptional invariants if $n$ is odd). It is also shown in [BEGr] that there are no exceptional invariants in Fefferman's original problem pertaining to $\mathrm{CR}$ geometry.

In this paper, following work of Gover [Go2] on the exceptional invariants connected with projective structures, we define basic exceptional invariants of $\mathscr{H}_{k}$ and $\mathscr{K}$ (see Propositions 3.1 and 4.1). Our main result is that in both cases, every exceptional invariant is a linear combinations of these (Theorems 3.2 and 4.4). For $\mathscr{K}$, we show also that all the basic exceptional invariants are zero when $n$ is not a multiple of 4 , and so in these cases, as for $n$ odd, all invariants of $\mathscr{K}$ are Weyl invariants. The basic exceptional invariants can be written down quite explicitly, and there are only a finite number in any dimension. This completes the work of [BEGr], in that we now have a means of listing all the invariants of these modules.

Our notation is almost entirely as in [BEGr]. We have tried to give a selfcontained treatment, but if we have erred on the side of brevity, we refer the reader to $[\mathrm{BEGr}]$ for clarification.

\section{Definitions}

For $n \geq 2$, let $W$ denote $\mathbb{R}^{n+2}$ with coordinates

$$
X^{I}=\left(\begin{array}{c}
X^{0} \\
X^{i} \\
X^{\infty}
\end{array}\right), \quad i=1, \ldots, n,
$$

and let $\mathscr{Q}$ denote the null cone of the quadratic form $\tilde{g}$ given by

$$
\tilde{g}_{I J} X^{I} X^{J}=2 X^{0} X^{\infty}+g_{i j} X^{i} X^{j}
$$

where $\left(g_{i j}\right)$ is a positive definite quadratic form on $\mathbb{R}^{n}$. The quadratic form $\tilde{g}$ provides an isomorphism of $W$ with its dual $W^{*}$ which we indicate by the use of "*", or by "raising and lowering indices" in the usual way. We use $\partial_{I}$ to denote the coordinate derivative $\partial / \partial X^{I}$. Let $e_{0} \in \mathscr{Q}$ denote the point with coordinates

$$
e_{0}^{I}=\left(\begin{array}{c}
1 \\
0 \\
\vdots \\
0
\end{array}\right),
$$

and let $P$ denote the parabolic subgroup of the identity-connected component $G$ of $\mathrm{O}(\tilde{g})$ defined by

$$
P=\left\{p \in G: p e_{0}=\lambda e_{0}, \text { for some } \lambda>0\right\} .
$$

Let $\sigma_{q}$ denote the 1-dimensional representation of $P$ where the element $p$ in (1) above acts by $\lambda^{-q}$.

We write $\mathscr{E}(k)$ for the $(\mathfrak{g}, P)$-module of jets at $e_{0}$ of functions on $W$ homogeneous of degree $k$ and $\mathscr{F}(k)$ for the $(\mathfrak{g}, P)$-module of jets at $e_{0}$ of 
functions on $\mathscr{Q}$ homogeneous of degree $k$. Since all jets to which we will refer are jets at $e_{0}$, we will omit the reference point henceforth. Jets are of functions on $W$ unless explicitly stated to be of functions on $\mathscr{Q}$.

We can regard the coordinates $X^{I}$ as being the components of the (homogeneity 1 ) identity function $X$ on $W$. The tensor $\tilde{g}$, its inverse $\tilde{g}^{-1}$ and the associated volume form $\tilde{\epsilon}$ all define constant fields on $W$ and hence constant jets. We abuse notation by relying on context to determine when we are thinking of these objects as jets.

Evaluation at $e_{0}$ yields a $P$-equivariant evaluation map

$$
\text { Eval : } \mathscr{E}(k) \rightarrow \sigma_{k},
$$

and we use the same notation for the evaluation $\mathscr{F}(k) \rightarrow \sigma_{k}$, and to the obvious generalizations to tensor-valued fields. We define $e \in W \otimes \sigma_{1}$ by

$$
e=\operatorname{Eval}(X) \text {. }
$$

Let $\Delta$ denote the (indefinite) Laplacian $\tilde{g}^{I J} \partial_{I} \partial_{J}$. (We use the summation convention throughout.) The operator $D$ is defined on jets of functions or tensor fields homogeneous of degree $s$ on $\mathscr{Q}$, where $n+2 s \neq 2$, by

$$
D_{I} f=\left.\left(\partial_{I} f-\frac{X_{I} \Delta f}{(n+2 s-2)}\right)\right|_{\mathscr{Q}},
$$

where an extension of $f$ off $\mathscr{Q}$ has been chosen-the result is independent of the choice. If $f \in \mathscr{F}(r)$ with $n+2 r \neq 0$, then

$$
D_{I}\left(X^{I} f\right)=\frac{(n+2 r+2)(n+r)}{n+2 r} f,
$$

and the same applies if $f$ has indices.

Choose a point $B \in W^{*}$ with $B\left(e_{0}\right) \neq 0$. Then

$$
\xi=\frac{B}{B(X)}
$$

defines a jet of homogeneity -1 taking values in $W^{*}$ and satisfying $\xi(X)=1$. Define a jet $\eta$ taking values in $\Lambda^{n} W$ and of homogeneity zero by

$$
\eta=\xi\lrcorner \tilde{\epsilon}_{0},
$$

where $\tilde{\epsilon}_{0}$ is the jet $\left.X^{*}\right\lrcorner \tilde{\epsilon}$. It is easy to check (using $\left.g_{I J} X^{I} X^{J}\right|_{\mathscr{Q}}=0$ ) that

$$
\left.\tilde{\epsilon}_{0}\right|_{\mathscr{Q}}=\left.(X \wedge \eta)\right|_{\mathscr{Q}} \text {. }
$$

We need to know how $\eta$ depends on the choice of $B$. Let $\hat{\xi}$ and $\hat{\eta}$ be the quantities defined as above, but starting with a different point $\hat{B} \in W^{*}$. A short calculation shows that there exists a jet $\rho$ taking values in $\Lambda^{n-1} W$ such that

$$
\left.\hat{\eta}\right|_{\mathscr{Q}}=\left.(\eta+X \wedge \rho)\right|_{\mathscr{Q}} \text {. }
$$

2.1. The scalar $P$-modules. Let $\mathscr{H}(k)$ denote the $(\mathfrak{g}, P)$-submodule of $\mathscr{E}(k)$ consisting of those elements which are harmonic with respect to $\Delta$. The harmonic polynomials in $\mathscr{H}(k)$ form a $(\mathfrak{g}, P)$-submodule, and we denote by $\mathscr{H}_{k}$ the corresponding quotient. As a $P$-module, $\mathscr{H}_{k}$ can be regarded as those jets that vanish to order $k+1$ at $e_{0}$, and then as a $P$-module $\mathscr{H}(k)$ is a direct 
sum of the harmonic polynomials with $\mathscr{H}_{k}$. Algebraically, elements of $\mathscr{H}_{k}$ are given by lists of tensors. In fact ([BEGr, Proposition 1.2], following [EG]):

$$
\mathscr{H}_{k}=\left\{\begin{array}{l}
\left(T^{(k+1)}, T^{(k+2)}, \ldots\right): T^{(l)} \in \bigodot_{0}^{l} W^{*} \otimes \sigma_{k-l}, \\
\left.e\lrcorner T^{(l+1)}=(k-l) T^{(l)} \text { for } l>k \text { and } e\right\lrcorner T^{(k+1)}=0
\end{array}\right\},
$$

where the symbol " $\bigodot_{0}^{l}$ " denotes the trace-free part of the $l$-fold symmetric tensor product. Given a jet $f$, the isomorphism is obtained by setting

$$
T^{(l)}=\operatorname{Eval}\left(\partial^{l} f\right), \quad l \geq k+1,
$$

where " $\partial$ " denotes the $l$-fold derivative.

2.2. The curvature $P$-module. For $l \geq 0$ and $n \geq 3$ we denote by $W^{(l)}$ the $G$-submodule of $\otimes^{l+4} W^{*}$ consisting of totally trace-free tensors enjoying the following symmetries

$$
\begin{gathered}
T_{I J K L, A B \cdots D}=T_{[I J][K L],(A B \cdots D),} \\
T_{[I J K] L, A B \cdots D}=0, \quad T_{I J[K L, A] B \cdots D}=0,
\end{gathered}
$$

where we use parentheses and square brackets to denote symmetrization and antisymmetrization respectively. The comma is merely a marker separating the first four indices from the remaining $l$.

Let $\mathscr{K}$ denote the $P$-module of jets of functions $f$ on $W$ homogeneous of degree -2 , taking values in $W^{(0)}$ and satisfying

$$
X^{L} f_{I J K L}=0, \quad \partial_{[H} f_{I J] K L}=0
$$

to all orders. As a $P$-module ([BEGr, Proposition 4.1]),

$$
\mathscr{K}=\left\{\begin{array}{l}
\left(T^{(0)}, T^{(1)}, \ldots\right): T^{(l)} \in W^{(l)} \otimes \sigma_{-l-2}, \\
e^{L} T_{I J K L, A B \cdots D}^{(l+1)}=-(l+1) T_{I J K(A, B \cdots D)}^{(l)} \text { for } l \geq 0, e^{L} T_{I J K L}^{(0)}=0
\end{array}\right\},
$$

with the correspondence being given by $T^{(l)}=\operatorname{Eval}\left(\partial^{l} f\right)$.

We will move freely between thinking of elements of $\mathscr{H}_{k}$ or $\mathscr{K}$ as lists of tensors and thinking of them as jets.

2.3. Weyl invariants. An odd Weyl invariant of $\mathscr{H}_{k}$ or $\mathscr{K}$ of degree $d$ is a linear combination of complete contractions each of which is of one of the forms

$$
\begin{aligned}
& \operatorname{contr}\left(\tilde{\epsilon} \otimes T^{\left(l_{1}\right)} \otimes T^{\left(l_{2}\right)} \otimes \cdots \otimes T^{\left(l_{d}\right)} \otimes \tilde{g}^{-1} \otimes \cdots \otimes \tilde{g}^{-1}\right), \\
& \operatorname{contr}\left(\tilde{\epsilon}_{0} \otimes T^{\left(l_{1}\right)} \otimes T^{\left(l_{2}\right)} \otimes \cdots \otimes T^{\left(l_{d}\right)} \otimes \tilde{g}^{-1} \otimes \cdots \otimes \tilde{g}^{-1}\right)
\end{aligned}
$$

and each taking values in $\sigma_{q}$ for some fixed $q$. In the case of $\mathscr{H}_{k}$, we have $l_{i} \geq k+1$, and for $\mathscr{K}$ we have $l_{i} \geq 0$. Note that we use the same notation $\tilde{\epsilon}_{0}$ for the jet $\left.\tilde{\epsilon}_{0}=X^{*}\right\lrcorner \tilde{\epsilon}$, as in (4), and for its image under evaluation at $e_{0}$, which is $e^{*} \downarrow \tilde{\epsilon}$ as immediately above. It should always be clear from context which is meant.

An invariant which is not a Weyl invariant is exceptional. An odd invariant of $\mathscr{H}_{k}$ is exceptional if and only if it is of degree $n$ and an odd invariant of $\mathscr{K}$ is exceptional if and only if it is of degree $n / 2$ [BEGr, Theorems $1.7,2.8,4.2 \&$ 5.3]. By elementary symmetry arguments, there are no non-zero odd invariants of $\mathscr{U}_{k}$ of degree $<n$ nor of $\mathscr{K}$ of degree $<n / 2$ [BEGr]. (There is a similar 
definition of even Weyl invariant-there are no even exceptional invariants of either $\mathscr{H}_{k}$ or $\mathscr{K}$.)

\section{THE SCALAR CASE}

Let $B, \eta$ be as in $\S 2$.

Proposition 3.1. Let $f \in \mathscr{H}_{k}$, and denote by $\partial^{k+1} f$ the (tensor-valued) jet obtained by $(k+1)$-fold coordinate differentiation of $f$. Let $L$ be a partial contraction of the form

$$
\operatorname{partcontr}(\eta \otimes \underbrace{\partial^{k+1} f \otimes \partial^{k+1} f \otimes \cdots \otimes \partial^{k+1} f}_{n} \otimes \tilde{g}^{-1} \otimes \cdots \otimes \tilde{g}^{-1}),
$$

taking values in $\otimes^{l} W \otimes \sigma_{-n}$ for $l \geq 0$ and such that all the indices of $\eta$ are contracted. Then

$$
I=\operatorname{Eval}(\underbrace{D_{I} \cdots D_{K}}_{l} L^{I \cdots K})
$$

is independent of the choice of $B$ and is an odd invariant of $\mathscr{H}_{k}$ of degree $n$. We call such invariants basic exceptional.

Proof. The indices of $\eta$ are necessarily contracted into the $\partial^{k+1} f$ 's. Since $X\lrcorner \partial^{k+1} f=0$ by Euler's equation, it follows from (6) that $\left.L\right|_{\mathscr{Q}}$ and hence $I$ are independent of the choice of $B$, and therefore $I$ is an invariant.

To obtain an expression for $I$, one uses the definition of $D$ in (2) and calculates using $\partial_{A} \xi_{B}=-\xi_{A} \xi_{B}$. The end result, having simplified also with the linking relations in (7), is that $I$ is a linear combination of complete contractions of $\eta, \tilde{g}^{-1}, \xi$ and derivatives of $f$. It is straightforward to check explicitly for particular small values of $k$ and $n$ that there exist non-zero examples of basic exceptional invariants and that, in particular, there exist examples which depend non-trivially on the $T^{(l)}$ for $l>k+1$. The analogous result for the curvature case is discussed in more detail in the following sections.

Our main result in the scalar case is:

Theorem 3.2. Every exceptional invariant of $\mathscr{H}_{k}$ is a linear combination of basic exceptional invariants.

Our starting point in proving the theorem is the same as that used in [BEGr] to show that odd invariants of degree $>n$ are Weyl invariants. Combining Proposition 2.5 and Lemma 2.4 of [BEGr] and specializing to the case of odd invariants of degree $n$, we obtain:

Proposition 3.3. Let $I: \mathscr{H}_{k} \rightarrow \sigma_{q}$ be an odd invariant of degree $n$. Then there exists a (g, P)-equivariant mapping $\tilde{I}: \mathscr{H}_{k} \rightarrow \mathscr{F}(q)$ with $\operatorname{Eval}(\tilde{I})=I$ and $a$ linear combination $\widetilde{C}$ of partial contractions of the tensor-valued jets $\partial^{l} f(l \geq$ $k+1), X, \tilde{g}^{-1}$ and $\tilde{\epsilon}_{0}$ such that as jets on $\mathscr{Q}$,

$$
\tilde{C}^{A B \cdots E}=X^{A} X^{B} \cdots X^{E} \tilde{I},
$$

not every partial contraction in $\widetilde{C}$ contains an $X$, and $m \leq 1-n-q$. 
Lemma 3.4. Let $I$ and $\widetilde{C}$ be as in Proposition 3.3, and let I be non-zero. Then $m=1-n-q$.

Proof. Equation (8) is between jets of homogeneity $\leq 1-n$, and so $D$ can be applied $m$ times to both sides. (Recall from (2) that $D$ can be applied to jets of homogeneity $s$ provided $n+2 s \neq 2$.) Evaluating at $e_{0}$, we obtain

$$
\operatorname{Eval}\left(D_{A} D_{B} \cdots D_{E} \widetilde{C}^{A B \cdots E}\right)=\operatorname{Eval}\left(D_{A} D_{B} \cdots D_{E}\left(X^{A} X^{B} \cdots X^{E} \tilde{I}\right)\right)
$$

Suppose $m<1-n-q$. Applying (3) $m$ times with $r$ taking the values $q+$ $m-1, q+m-2, \ldots, q$, we see that the right-hand side is a non-zero multiple of $I$. Expanding using the definition (2) of $D$, the left-hand side is seen to be a Weyl invariant (cf. [BEGr, Proof of Theorem 2.8]), but every odd Weyl invariant of degree $n$ is zero, and hence $I$ is zero, contrary to hypothesis.

Proof of Theorem 3.2. Let $I$ and $\widetilde{C}$ be as in Proposition 3.3, with $I$ non-zero, so that by Lemma 3.4 , we may assume $m=1-n-q$. Write $\widetilde{C}$ as a sum

$$
\widetilde{C}=\widetilde{C}_{0}+\widetilde{C}_{x}
$$

where $\widetilde{C}_{0}$ consists of the sum of those terms containing no $X$ 's (here, as in Proposition 3.3, we assume that any contracted $X$ 's have been eliminated using Euler's equation). By taking the symmetric parts if necessary, we can assume that $\widetilde{C}_{0}$ and $\widetilde{C}_{x}$ are separately symmetric. Observe that $\widetilde{C}$ has homogeneity $1-n, \tilde{\epsilon}_{0}$ has homogeneity 1 , and $\partial^{l} f$ has homogeneity $k-l$. Since $\widetilde{C}$ has degree $n$, each term of $\widetilde{C}_{0}$ must contain exactly $n$ occurrences of $\partial^{k+1} f$, exactly $n$ indices of $\tilde{\epsilon}_{0}$ are contracted into these derivatives of $f$, and no other derivatives of $f$ occur.

Let $\widetilde{C}_{\eta}$ be obtained from $\widetilde{C}_{0}$ by replacing $\tilde{\epsilon}_{0}^{J K \ldots M}$ with $X^{[J} \eta^{K \ldots M]}$ and expanding the antisymmetrization. On $\mathscr{Q}$, we have $\widetilde{C}_{\eta}=\widetilde{C}_{0}$ by (5). All the terms in $\widetilde{C}_{\eta}$ where the $X$ does not contribute a free (uncontracted) index vanish because $X\lrcorner \partial^{k+1} f=0$. It follows that

$$
\tilde{C}_{\eta}^{A B \ldots E}=X^{(A} \tilde{E}_{\eta}^{B \ldots E)}
$$

where $\tilde{E}_{\eta}$ is a linear combination of partial contractions of the form of $L$ in Proposition 3.1 .

Since every term in $\widetilde{C}_{x}$ contains an $X$ with a free index, we may also write

$$
\tilde{C}_{x}^{A B \ldots E}=X^{(A} \tilde{E}_{x}^{B \ldots E)}
$$

for some linear combination of partial contraction $\tilde{E}_{x}$. Substituting into (8) and canceling an $X$ throughout yields

$$
\tilde{E}_{\eta}^{B \cdots E}+\tilde{E}_{x}^{B \ldots E}=\overbrace{X^{B} \ldots X^{E}}^{-n-q} \tilde{I} .
$$

We can apply $D_{B} \ldots D_{E}$ to both sides of this equation and evaluate at $e_{0}$ :

$$
\operatorname{Eval}\left(D_{B} \ldots D_{E}\left(X^{B} \ldots X^{E} \tilde{I}\right)\right)
$$


is a non-zero multiple of $I$ (by repeated use of (3)), and

$$
\operatorname{Eval}\left(D_{B} \ldots D_{E} \tilde{E}_{\eta}^{B \ldots E}\right)
$$

is a linear combination of basic exceptional invariants. The remaining term is an invariant (being the difference of two invariants), and since it is an odd Weyl invariant of degree $n$, it must be zero.

\section{THE CURVATURE CASE}

In this section, let $n$ be even (otherwise there are no exceptional invariants of $\mathscr{K}$ ). The proof of the following proposition is analogous to that of Proposition 3.1

Proposition 4.1. Let $f \in \mathscr{K}$, and let $L$ be a partial contraction of the form

$$
\operatorname{partcontr}(\eta \otimes \underbrace{f \otimes f \otimes \cdots \otimes f}_{n / 2} \otimes \tilde{g}^{-1} \otimes \cdots \otimes \tilde{g}^{-1}),
$$

taking values in $\otimes^{l} W \otimes \sigma_{-n}$ for $l \geq 0$ and such that all the indices of $\eta$ are contracted. Then

$$
I=\operatorname{Eval}(\underbrace{D_{I} \cdots D_{K}}_{l} L^{I \cdots K})
$$

is independent of the choice of $B$ and is an odd invariant of $\mathscr{K}$ of degree $n$. We call such invariants basic exceptional.

The question was raised in [BEGr] as to whether all exceptional invariants of $\mathscr{K}$ depend only on $T^{(0)}$. We provide an example in section 5 of a basic exceptional invariant of $\mathscr{K}$ for $n=4$, which depends non-trivially on $T^{(l)}$ for $l>0$.

We will show that all exceptional invariants of $\mathscr{K}$ are linear combinations of basic exceptional invariants, and also that every basic exceptional invariant is zero when $n \equiv 2(\bmod 4)$. We start with the second of these claims, the proof of which requires the following lemma.

Lemma 4.2. Let $f \in W^{(0)}$, and let $S$ be a partial contraction of $\eta, f$ and $\tilde{g}^{-1}$ of the form

$$
\begin{aligned}
& S_{A B}^{I_{2 m+1} I_{2 m+2} \ldots I_{n}} \\
& \quad=\eta^{I_{1} \ldots I_{n}} f_{I_{1} I_{2} A K_{1}} f_{I_{3} I_{4} L_{1} K_{2}} f_{I_{5} I_{6} L_{2} K_{3}} \cdots f_{I_{2 m-1} I_{2 m} L_{m-1} B} \tilde{g}^{K_{1} L_{1}} \cdots \tilde{g}^{K_{m-1} L_{m-1},}
\end{aligned}
$$

where $2 m \leq n$. If $m \equiv 1(\bmod 2)$, then

$$
S_{(A B)}^{I_{2 m+1} I_{2 m+2} \ldots I_{n}}=0 .
$$

Proof. Observe that $\eta^{I_{1} \ldots I_{n}}$ is unchanged by any permutation of the $n / 2$ index pairs $\left(I_{1} I_{2}\right), \ldots,\left(I_{n-1} I_{n}\right)$. Using this and the fact that each $f$ is antisymmetric in its third and fourth indices the result follows by straightforward calculation.

Proposition 4.3. If $n \equiv 2(\bmod 4)$, then every basic exceptional invariant of $\mathscr{K}$ is zero. 
Proof. Let $n \equiv 2(\bmod 4)$. We will show that any $L$ constructed as in Proposition 4.1 is zero or vanishes when symmetrized. Since the operator $D_{I} \ldots D_{K}$ is symmetric in the indices $I \ldots K$, this suffices to prove the proposition.

In the expression (12) for $L$, all the indices of $\eta$ are contracted, and if more than 2 such indices are contracted into the same $f$, the result vanishes by the symmetries of $f$. The result of contracting two indices of $\eta$ into $f$ is independent (up to a non-zero constant) of the indices chosen. We may assume therefore that the first two indices of each $f$ are contracted into $\eta$.

In the expression (12), we will say that two $f$ 's are contracted if there is a $\tilde{g}^{-1}$ which has one index contracted with each of them. Consider the smallest equivalence relation on the set of $f$ 's with the property that contracted $f$ 's are related (i.e., two $f$ 's are in the same equivalence class if they are joined by a chain of contractions). Since $n / 2$ is odd, at least one of these equivalence classes must have an odd number of $f$ 's. Consider such an equivalence class of $f$ 's, and note that it must occur as an expression like that of Lemma 4.2, with the indices $A, B$ either contracted with $\tilde{g}^{A B}$ or both raised with $\tilde{g}^{-1}$ and appearing as free indices in $L$. In the first case, Lemma 4.2 gives that $L=0$, and in the second case, it gives that $L$ will vanish when symmetrized.

Theorem 4.4. If $n \not \equiv 0(\bmod 4)$, then $\mathscr{K}$ has no exceptional invariants. If $n \equiv 0(\bmod 4)$, then every exceptional invariant of $\mathscr{K}$ is a linear combination of basic exceptional invariants.

Proof. For $n$ odd, this is proved in [BEGr]. Given Proposition 4.3, it suffices to show that for all even $n$, every invariant is a linear combination of basic exceptional invariants. The proof of this follows the proof of Theorem 3.2, using analogues of Proposition 3.3 and Lemma 3.4.

\section{Closing Remarks}

A basic exceptional invariant of $\mathscr{K}$ for $n=4$ is given by

$$
J=\operatorname{Eval}\left(D_{I} D_{J}\left(\eta^{A B C D} f_{A B}^{I K} f_{C D K}{ }^{J}\right)\right),
$$

where we have used $\tilde{g}$ to raise some indices compared with the standard form given by Proposition 4.1.

Given a list of tensors $\left(u^{(l)}, l=0,1,2, \ldots\right)$ on $\mathbb{R}^{n}$, where each $u^{(l)}$ has the symmetries of the corresponding $T^{(l)}$ and is trace-free with respect to $g$, one can construct an element of $\mathscr{K}$ such that $T_{i j k l, a b \cdots d}^{(l)}=u_{i j k l, a b \cdots d}^{(l)}$ and all the $\infty$-components of the $T^{(l)}$ 's vanish (see [BEGr, $\left.\S 5\right]$ ). On such elements, it can be shown by straightforward calculation that the invariant $J$ is equal to

$$
-\frac{3}{16} \epsilon^{a b c d} u_{a b i j k}^{(1)} u_{c d}^{(1) i j k}
$$

This does not vanish identically, and so the invariant depends non-trivially on $T^{(l)}$ for $l>0$.

The connection between the invariant theory of $\mathscr{K}$ and the problem of invariants of conformal structures is not straightforward (see [BEGr] for an outline and [FG2] for details), but it was the existence of this invariant of $\mathscr{K}$ which led Eastwood and the authors to conjecture the existence of, and thus construct, the odd invariant of 4-dimensional conformal structures given in [BEGo]. 


\section{REFERENCES}

[BEGo] T.N. Bailey, M.G. Eastwood, and A.R. Gover, The Thomas structure bundle for conformal, projective and related structures, Rocky Mountain J. Math. (to appear).

[BEGr] T.N. Bailey, M.G. Eastwood, and C.R. Graham, Invariant theory for conformal and CR geometry, Ann. of Math. (2) 139 (1994), 491-552.

[EG] M.G. Eastwood and C.R. Graham, Invariants of conformal densities, Duke Math. J. 63 (1991), 633-671.

[F] C. Fefferman Parabolic invariant theory in complex analysis, Adv. Math. 31 (1979), 131262.

[FG2] C. Fefferman and C.R. Graham (in preparation).

[Go] A.R. Gover, Invariants on projective space, J. Amer. Math. Soc. 7 (1994), 145-158.

[Go2] Invariant theory for a parabolic subgroup of $S L(n+1, \mathbb{R})$, Proc. Amer. Math. Soc. 123 (1995), 1543-1553.

[Gr] C.R. Graham, Invariant theory of parabolic geometries, Complex Geometry (Komatsu and Sakane, eds.), Marcel Dekker, New York, 1993.

Department of Mathematics, University of Edinburgh, Scotland EH9 3JZ

E-mail address: tnbailey@edinburgh.ac.uk

Department of Pure Mathematics, University of Adelaide, GPO Box 498, Adelaide, South Australia 5001

Current address: School of Mathematics, QUT Gardens Point Campus, Brisbane, Queensland 2434, Australia

E-mail address: r.gover@qut.edu.au 